\title{
Long-term effects of chondrospheres on cartilage lesions in an autologous chondrocyte implantation model as investigated in the SCID mouse model
}

\author{
T. SCHUBERT ${ }^{1}$, S. ANDERS ${ }^{2}$, E. NEUMANN ${ }^{3,4}$, J. SCHÖLMERICH ${ }^{3}$, F. HOFSTÄDTER ${ }^{1}$, \\ J. GRIFKA ${ }^{2}$, U. MÜLLER-LADNER ${ }^{3,4}$, J. LIBERA ${ }^{5}$ and J. SCHEDEL ${ }^{3,6}$
}

\begin{abstract}
Departments of ${ }^{1}$ Pathology; ${ }^{2}$ Orthopedic Surgery; ${ }^{3}$ Internal Medicine I, University Hospital of Regensburg, Regensburg;
${ }^{4}$ Department of Internal Medicine and Rheumatology, Kerckhoff Clinic and University of Giessen, Giessen;

${ }^{5}$ Co.don Research Center, Teltow; ${ }^{6}$ Department of Internal Medicine II,

University Hospital of Tuebingen, Tuebingen, Germany
\end{abstract}

Received November 18, 2008; Accepted January 5, 2009

DOI: 10.3892/ijmm_00000151

\begin{abstract}
Microtraumata often lead to articular cartilage lesions. Due to the bradytrophic character of hyaline cartilage, these lesions are hardly repaired by the organism. Autologous chondrocyte implantation (ACI) was established for restoring isolated structural cartilage defects in knee joints. However, results are not always convincing. Human chondrocytes from patients undergoing total knee arthroplasty were cultured in monolayer followed by condensing single chondrocytes to spheroids (chondrospheres ${ }^{\circledR}$ ). The integrative capacity of chondrospheres was examined by implanting them into lesions in human articular cartilage specimens and co-implanting them into SCID mice. Mice were sacrificed after 4, 12 and 24 weeks. HE and safranin O staining as well as immunohistochemistry using anti-S100, anti-collagen I and II antibodies were performed and analyzed using semiquantitative scores. Integration of the chondrospheres with the (native) cartilage matrix was analyzed by determining the percentage of adhering surface. With respect to long-term stability, the chondrocytes within chondrospheres showed a typical chondrocytic morphology. Immunohistochemically, a high collagen II production was detected. Over a time period of 24 weeks, an increasing content of collagen type II, glycosaminoglycans and collagenous fibers were found. Importantly, the newly synthesized cartilaginous matrix integrated continuously with the native cartilage lesion border. In conclusion, the presented data demonstrate that chondrospheres are able to restore and conserve their phenotype for at least 24 weeks under in vivo
\end{abstract}

Correspondence to: Dr Jörg Schedel, Department of Internal Medicine II, Division of Rheumatology and Clinical Immunology, University Hospital of Tuebingen, D-72076 Tuebingen, Germany

E-mail: joerg.schedel@med.uni-tuebingen.de

Key words: chondrosphere, cartilage lesion, autologous chondrocyte implantation, severe combined immunodeficiency mouse model conditions. Moreover, chondrospheres adhere to full-thickness cartilage defects and appear to produce a cartilaginous extracellular matrix which fuses with native cartilage thus generating an autologous cartilage-like repair tissue.

\section{Introduction}

Cartilage lesions can be a consequence of etiologically and pathogenetically different primary and secondary disease entities of the joint. The lack of healing, both partial and fullthickness defects, is due to the insufficient regenerative capability of hyaline cartilage to synthesize cartilage-specific extracellular matrix molecules necessary for integration (1).

Different surgical procedures have been implemented for the treatment of hyaline cartilage defects. These methods are mainly directed towards accessing the subchondral vasculature and bone marrow (2) either by abrasion of the cartilage and subchondral bone plate with powered burrs (3-5), drilling of the subchondral bone (6), or microfracturing (7). However, the fibrocartilage-like reparative tissue that results from these techniques lacks the composition, structure, and mechanical properties of normal cartilage and the long-term clinical outcome is unpredictable (8). Patients treated by these techniques often experience only short-term pain relief but develop progressive osteoarthritic symptoms when the reparative tissue disintegrates (5). Alternatively, various surgical procedures were developed to deliver autologous chondrogenic precursor cells to the cartilage defect in the form of cell suspensions prepared by expanding cells originating from cartilage biopsies (9), precursor cells derived from the periosteum (10), or the perichondrium (11), with the latter eventually undergoing terminal differentiation to chondrocytes in situ.

Approaches to 'engineer' cartilage ('tissue engineering') were undertaken using cultivated de-differentiated autologous chondrocytes being implanted into the cartilage defect site (12). Though autologous chondrocyte implantation (ACI) has good clinical results in the long run (13), the questions of a sufficient attachment and integration of these cells in the cartilage lesion and the maintenance of their biomechanical properties for an extended period of time are unresolved. The implanted cells 
are insufficiently characterized with respect to viability, degree of differentiation, morphology, or production of extracellular matrix (14). Furthermore, covering the cartilage defect with a periosteal flap as effected in the original ACI procedure (9) represents a time- and skill-demanding invasive surgical procedure. To improve surgical treatment as a minimally invasive procedure, an in vitro engineered autologous chondrocytic tissue (chondrosphere ${ }^{\circledR}$ ) was developed. In this study, the integration as well as cartilage remodeling and maturation under in vivo conditions were analyzed in a model analogous to ACI using the SCID mouse co-implantation model (15).

\section{Materials and methods}

Chondrocyte culture and spheroid generation. Articular cartilage was obtained from macroscopically unworn human articular condyles from 5 voluntary patients undergoing total knee arthroplasty after written informed consent. Cartilage (60-100 mg) was minced and digested in a $50 \mathrm{ml}$ Falcon tube using 20-25 U/mg collagenase type II (Biochrome, Germany) at $37^{\circ} \mathrm{C}$ for $8 \mathrm{~h}$ in a gyratory shaker $(110 \mathrm{r} /$ minute $)$. Isolated cells were washed and resuspended in culture medium with the addition of pooled human serum from different human volunteers $(n=6)$. No growth factors, cytokines or other supplements were added. Chondrocytes were seeded in culture flasks (Falcon, $75 \mathrm{~cm}^{2}$ ) and maintained at $37^{\circ} \mathrm{C}$ in a humidified atmosphere and $5 \% \mathrm{CO}_{2}$. Medium was changed twice per week. After reaching confluence the cells were trypsinized using trypsin-EDTA (PAA-Laboratories $\mathrm{GmbH}$, Germany) and cultured in larger flasks (Falcon, $225 \mathrm{~cm}^{2}$ ). For generation of spheroids, $2 \times 10^{5}$ cells of third passage chondrocytes were seeded in hydrogel-coated 96-well-plates (Fig. 1). After 1 week, four to twelve single spheroids were transferred into one well allowing coalescence of spheroids. After 2 weeks, fused spheroids were used for subcutaneous transplantation. Single spheroids were cultured for 3 weeks before transplantation.

Severe combined immunodeficiency (SCID) mouse model. The integrative capacity of the chondrospheres was investigated in the severe combined immunodeficiency (SCID) mouse coimplantation model (15). First, using a sharp spoon, small, nearly full-thickness, lesions of a $5 \mathrm{~mm}$ diameter and 3-4 mm depth were generated in fresh, healthy appearing human cartilage specimens which were obtained during total knee arthroplasty from 5 patients suffering osteoarthrosis at the Department of Orthopedics, University of Regensburg, Germany. All the patients gave written, informed consent to the study. Several chondrospheres (5-12, according to the maximum number of chondrospheres available) were put into the defects, and these constructs (in total 37) were implanted subcutaneously into SCID mice. Work, with respect to SCID mice, was approved by the local ethics committee of the Medical faculty of the University of Regensburg. The 14-week old SCID mice were obtained from a germ-free breeding colony (Charles River, Sulzfeld, Germany) and examined for macroscopic anomalies before and during surgery as well as for macro- and histopathologic abnormalities after sacrifice. The mice were anesthetized and a small incision was made in the left and right flank. After preparation of an appropriate

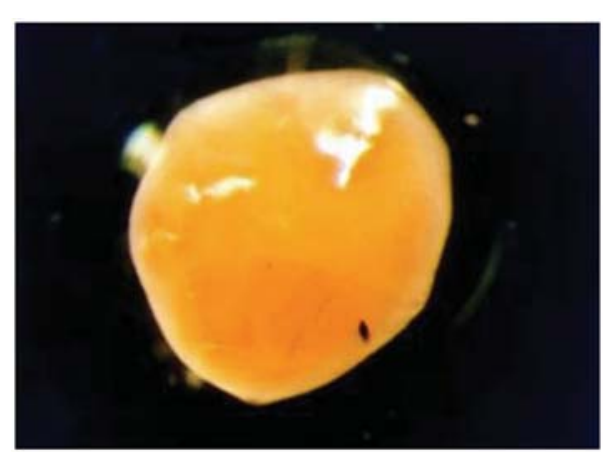

Figure 1. Chondrosphere ${ }^{\circledR}$ spheroids were generated by culturing $2 \times 10^{5}$ cells of third passage chondrocytes in hydrogel-coated 96 -well plates.

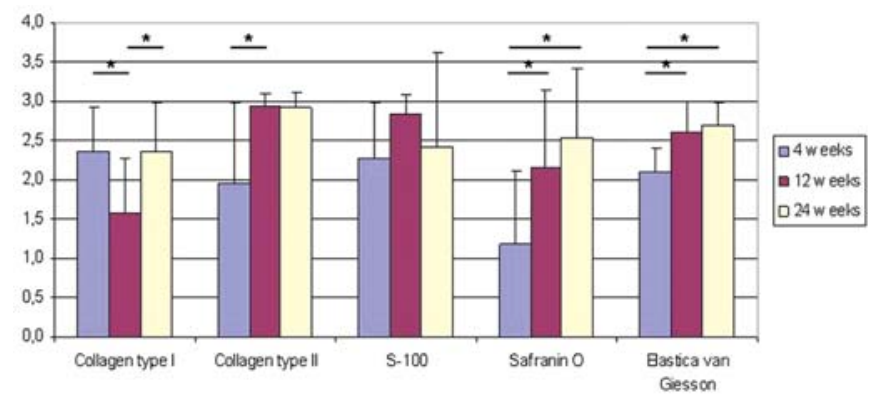

Figure 2. Production of extracellular matrix proteins by chondrospheres detected by immunohistochemistry using antibodies against collagen types I, II and $\mathrm{S} 100$, as well as histological staining with safranin $\mathrm{O}$ and Elastica van Giesson. The numbers of the experiments were 7,10 and 8 for weeks 4,12 and 24 , respectively. ${ }^{*} \mathrm{p}<0.05$.

cavum in the subcutaneous tissue, the constructs were implanted, and subsequently, the skin was sutured. After 4, 12 and 24 weeks, the mice were sacrificed and the implants removed. After decalcification in a saturated EDTA solution, the tissue was dehydrated, fixed in $4 \%$ buffered formalin and embedded in paraffin according to standard procedures.

Immunohistology and immunohistochemistry. Serial paraffin sections ( 3 to $5 \mu \mathrm{m}$ thickness) were cut through the center of the cartilage defect, and were mounted on 'Superfrost Plus' slides, heated for $20 \mathrm{~min}$ at $72^{\circ} \mathrm{C}$, deparaffinized and rehydrated. Following antigen retrieval in a citrate buffer at $\mathrm{pH} 7.3 \mathrm{in}$ a microwave for $30 \mathrm{~min}$ at $240 \mathrm{~W}$, the slides were rinsed and endogenous peroxidase activity was blocked using methanolic peroxide. The slides were rinsed again and primary monoclonal antibodies against collagen I, II (MP Biomedicals, LLC, Aurora, Ohio, USA; both dilutions $10 \mu \mathrm{g} / \mathrm{ml}$ ) and S100 (Dako, Hamburg, Germany, dilution 1:100) were applied. Slides for S100 immunohistochemical staining were incubated using a Ventana machine (Ventana Medical Systems, basic DAB detection kit, Ventana Medical Systems Inc., USA). The streptavidin-biotin-peroxidase method was used for visualization. The immunohistochemical staining procedures for collagen I and II were performed manually. After incubation with the primary antibody, the slides were rinsed and the biotinylated secondary antibody was applied at a dilution of 1:200. The streptavidin-biotin-peroxidase method was used for visualization resulting in brown staining of 

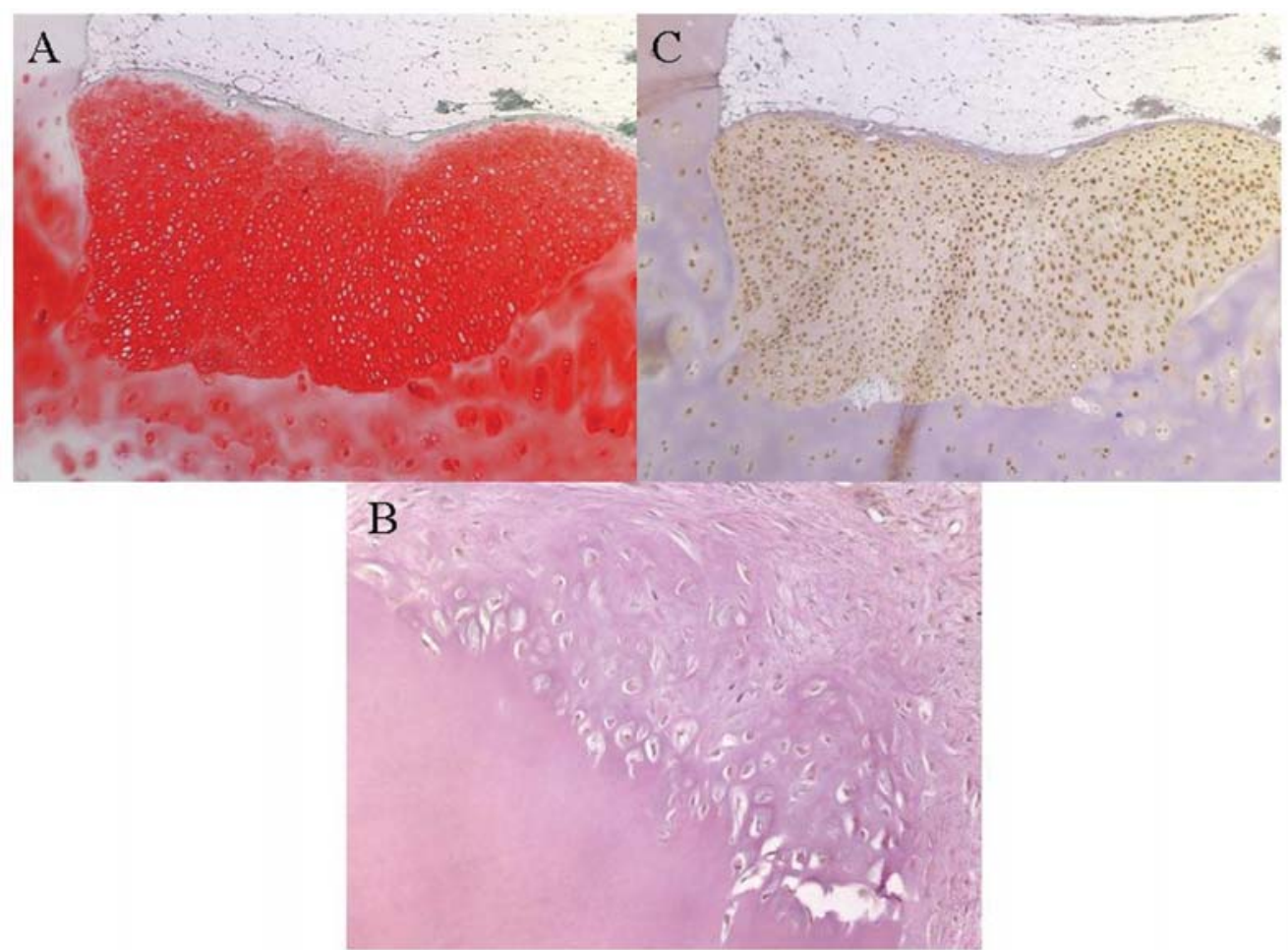

Figure 3. (A) Histological staining with safranin O, week 12 and (B) Elastica van Giesson, week 4 as well as (C) immunohistochemistry using antibodies against S100, week 12 in paraffin sections of chondrospheres implanted in cartilage lesions. Original magnifications A and C, x50; B, x200.

extracellular matrix, counterstaining was applied using hematoxylin. For negative controls, isotype-matched control antibodies were employed. Safranin O and Elastica van Giesson staining were performed according to standard procedures.

Histological evaluation. The sections were evaluated by a semiquantitative score. In respect to reactivity with $\mathrm{S} 100$, the following grading was applied, 0 , expression in $0-25 \%$ of cells; $1,26-50 \% ; 2,51-75 \% ; 3,76-100 \%$. In analogy, staining with safranin $\mathrm{O}$ and Elastica van Giesson as well as immunohistochemistry for collagen types I and II was scored semiquantitatively, 0 , no; 1 , weak; 2 , medium; and 3 , strong staining. As internal reference, the strength of the respective staining in the adjacent native cartilage or bone was quantified as 3 points representing the maximum score of expression. Similarly, chondrocyte morphology was evaluated using a score ranging from 0 , fibrocytic to 3 , chondrocytic appearance. Integration of the chondrospheres with the (native) cartilage matrix was defined as the percentage of the chondrosphere surface adhering to the cartilage lesion borders evaluated in 3 consecutive sections. As such, $100 \%$ integration would indicate coverage of the complete surface of the spheroid by the (native) cartilage borders which, however, was not feasible for geometrical reasons. The evaluation was performed by two independent investigators (T.S., J.S.), who were blinded to the point of time at which the mice were sacrified.

Statistical evaluation. Statistical analysis of histological and immunohistological semiquantitative data was performed using SPSS 13.0. Using the non-parametrical Mann-Whitney U-test p-values $<0.05$ were regarded as significant.

\section{Results}

Histomorphology of constructs after SCID mouse sacrifice. After 4, 12 and 24 weeks, 7/12, 10/13 and 8/12, respectively, constructs comprising chondrospheres within the artificially generated cartilage lesion were evaluated.

Production of hyaline cartilage-specific markers by chondrospheres. Using routine histology, a significant increase in the staining intensity of safranin $\mathrm{O}$ as indicator of glycosaminoglycan (GAG) content of the newly synthesized cartilage matrix was shown after 24 weeks when compared to 4 weeks $(\mathrm{p}=0.026)$. The safranin $\mathrm{O}$ staining intensity resembled the intensity of the surrounding hyaline cartilage which served as internal control (Figs. 2 and 3A). The intensity of staining for Elastica van Giesson representing collagenous fibers (regardless of type) started from a higher level than safranin $\mathrm{O}$ expression and also increased significantly over time $(\mathrm{p}=0.013$ after 24 weeks; Figs. 2 and 3B). S100 expression was detected at a medium level at 4 weeks after implantation and increased after 12 weeks $(\mathrm{p}=0.58)$. After 24 weeks no further upregulation of S100 was observed (Figs. 2 and 3C).

Using immunohistochemistry, an intense collagen II deposition was detected after 4 weeks which increased for up to 12 weeks ( $p=0.015)$, and after 24 weeks collagen type II expression levels were similar to the surrounding cartilage (Figs. 2, 4A and B). Collagen type I expression, on the other hand, was slightly higher after 4 weeks when compared to collagen type II expression (Fig. 4C), however, decreased within 12 weeks $(p=0.029)$ to again increase after 24 weeks ( $\mathrm{p}=0.028$; Figs. 2 and 4D). After 24 weeks collagen type II 


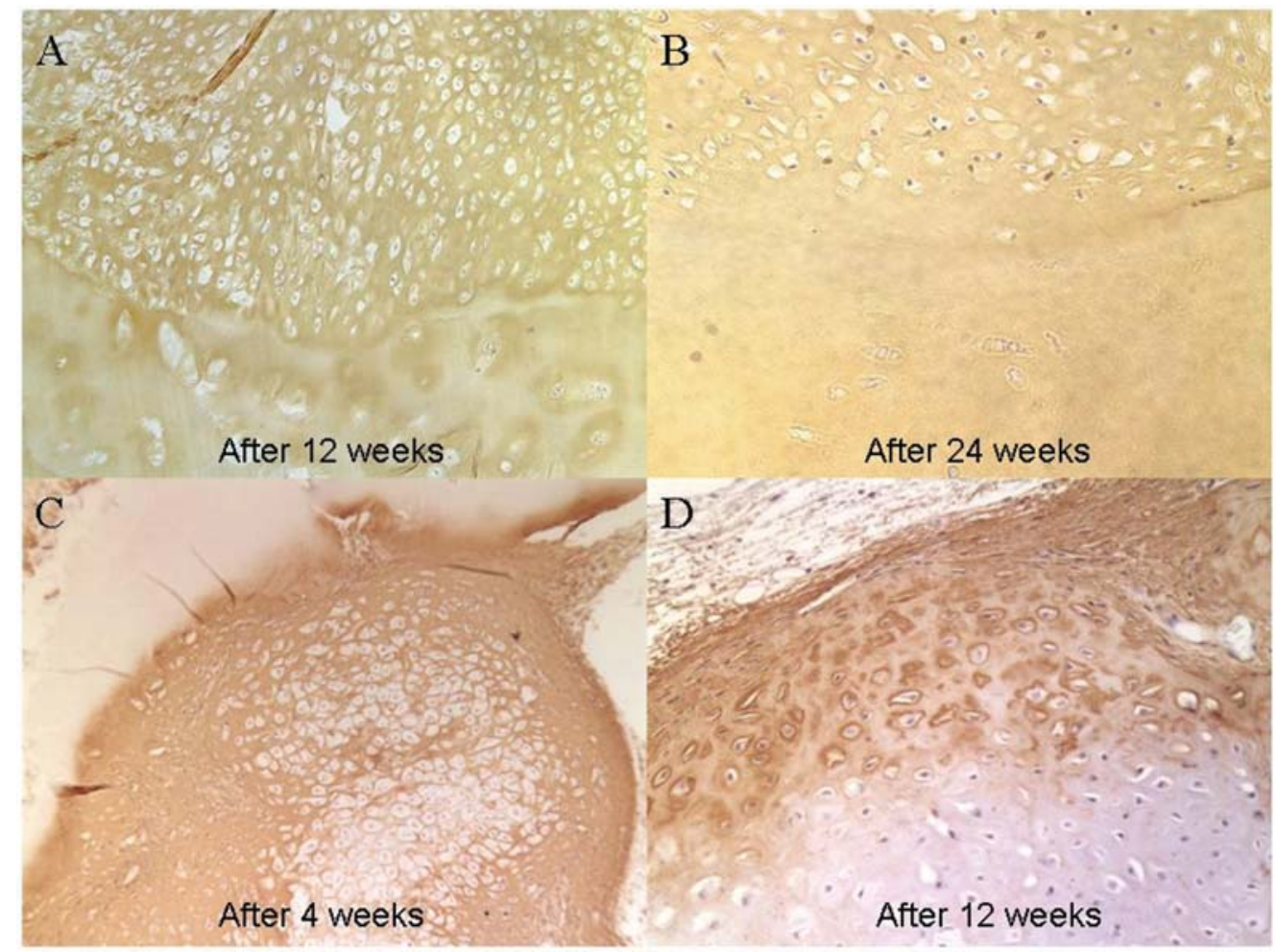

Figure 4. Immunohistochemistry using antibodies against collagen type II after 12 (A) and 24 (B) weeks, as well as against collagen type I after 4 (C) and 12 (D) weeks, respectively, in paraffin sections of chondrospheres implanted in cartilage lesions. Original magnifications A and C, x100; B and D, x200.
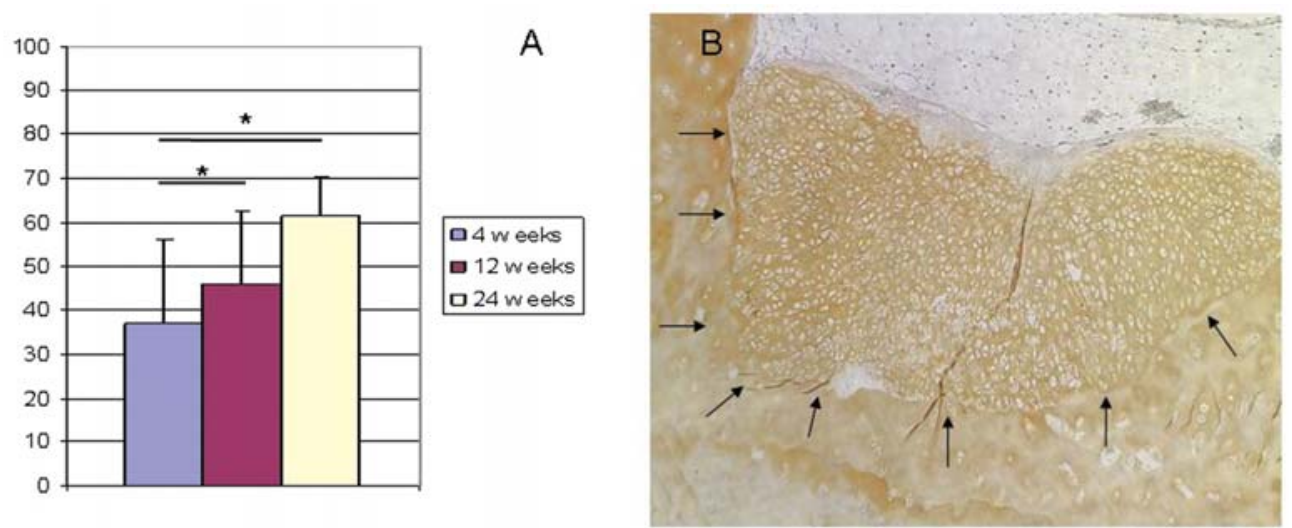

Figure 5. (A) Integration of newly synthesized extracellular matrix produced by chondrospheres with the cartilage lesion border. Integration was defined as the percentage of the chondrosphere surface adhering to the cartilage lesion borders. " $\mathrm{p}<0.05$. (B) Integration as visualized by collagen type II production of chondrospheres. Arrows indicate the site of integration. Original magnification x50.

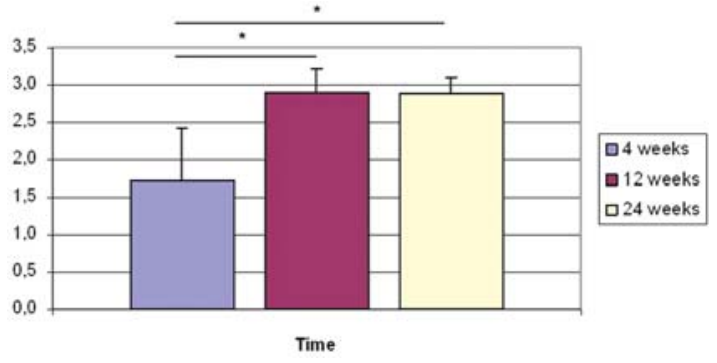

Figure 6. Change of chondrosphere morphology over time. 1, fibroblast-like phenotype; 3 , chondrocytic phenotype. ${ }^{*} \mathrm{p}<0.05$. expression was stronger than collagen type I expression reflecting the predominating hyaline cartilage-specific properties of the neo-generated matrix.

Integration of matrix produced by chondrospheres into native cartilage. Integration was defined as the percentage of the surface of the chondrosphere adhering to the cartilaginous substrate. Extensive integration of the cartilaginous matrix newly synthesized by the chondrospheres with the cartilage lesion borders was found after 4 weeks. Importantly, after 12 and 24 weeks, this integrative process continued and after 24 weeks, was significantly higher than after 4 weeks $(p=0.016$; Fig. 5A and B). 
Chondrocyte morphology within chondrospheres. After 4 weeks, about half of the chondrocytes within transplanted chondrospheres showed a typical chondrocytic morphological appearance (round cell shape with small round nucleus), others exhibited a rather fibrocytic phenotype (elongated cell body, oval nucleus). After 12 and 24 weeks, however, almost all chondrospheres were of typical chondrocytic morphology (Fig. 6).

\section{Discussion}

Cartilage lacks the ability to regenerate adequately after structural damage. Moreover, small cartilage lesions initiate a progressive degradation that eventually may lead to osteoarthritis. Autologous chondrocyte implantation (ACI) is widely used to regenerate focal traumatic cartilage lesions (16-18). However, the method is technically demanding due to the necessity of using a chondrocyte suspension and suturing a periosteal graft or a synthetic membrane over the cartilage defect. Therefore, a less complicated operative procedure is desirable for the biological treatment of cartilage lesions. The transplantation of an in vitro engineered solid cartilage-like tissue may provide an alternative to conventional ACI. The engineering of chondrocyte spheroids is one way to obtain grafts suited to this purpose (19). However, little is known about the capacity of spheroids to integrate, proliferate, differentiate and maintain a chondrocytic phenotype following their implantation into an articular lesion in humans in vivo. In order to study the capacity of chondrocyte spheroids ('chondrospheres' ${ }^{\circledR}$ ) to integrate into articular lesions in vivo, spheroid loaded human cartilage explants were investigated as an ACI analogon in the SCID mouse co-implantation model. We showed that chondrospheres were able to adhere and integrate into native hyaline cartilage. Proliferation and differentiation were paralleled by the production of cartilagespecific proteins which increased over time, and a preserved chondrocytic phenotype. These results support the finding of a study performed in which the authors used the same approach with chondrospheres which were transplanted into cartilage lesions in horse joints (20). In this study, newly synthesized extracellular matrix produced by ACI-grafted chondrospheres integrated sufficiently into the adjacent cartilage resulting in significantly better macroscopic and microscopic healing of the cartilage lesion when compared to untreated controls.

Since conclusions drawn from animal models are difficult to extrapolate for corresponding human conditions (21), in our study artificial defects were created within human cartilage explants which were used for subsequent chondrosphere adhesion. Co-implants of spheroids and human cartilage specimens were transplanted subcutaneously into the SCID mouse model which was originally designed for in vivo evaluation of the invasive properties of human synovial fibroblasts with respect to the co-implanted cartilage in rheumatoid arthritis (15). The approach to investigate the integrative capacity of chondrospheres in the in vivo SCID mouse coimplantation model is novel, and was chosen because in vitro experiments lack the respective specific, articular environment required for maintenance of chondrocyte metabolism. As an in vivo model, it combines the advantage of enabling an evaluation under in vivo conditions for an extended period of time, and at the same time, ease of use. To the best of our knowledge, the model presented here is the first ACI analogon model investigating human chondrocytes under in vivo conditions in SCID mice.

Most importantly, over time, the transplanted chondrospheres exhibited a significantly closer attachment and integration into the surrounding native cartilage thus meeting the essential requirement for tissue reconstruction. This integrative process was paralleled by a time-dependent significant increase in cartilage-specific collagen type II protein and GAG. The process of matrix maturation was underscored by the increasing production of S100. Equally, the amount of fibers detected by Elastica van Giesson staining increased over 24 weeks, representing the combined content of collagen types I and II fibers. On the other hand, collagen type I protein, often seen in regenerating fibro-cartilage-like tissue, was expressed in lower amounts as was collagen type II after 24 weeks. These findings are in line with a study published in which monolayer-expanded adult human articular chondrocytes were seeded into disks and were subsequently implanted subcutaneously in nude mice for up to 8 weeks (22). In contrast to freshly seeded scaffolds, the effects described in this study were only achieved after preculturing the chondrocytes in low density and after addition of differentiation medium for 2 weeks. Importantly, in our study, no growth factors, cytokines or other supplements were added to the transplanted chondrospheres. Nevertheless, the transplanted cells developed a hyaline cartilage-specific phenotype over time. Although the effect of 'native' transplanted chondrocytes was not compared with growth factor stimulated chondrocytes in our study, our results are encouraging in view of a study in which improved integration was achieved only by using a mixture of primary and TGF- $\beta$ producing chondrocytes (23).

Our results, therefore, suggest that a close contact with their (native) extracellular matrix is sufficient for chondrospheres to arrange themselves in three-dimensional cell aggregates in order to synthesize cartilage-specific proteins and matrix components and to deposit them in the intercellular space.

In ACI procedures periostal grafts are thought as essential both in animal models and humans (24) for different reasons: periostal flaps are able to cover the transplanted chondrocytes and retain them within the cartilaginous lesion (9). Furthermore, they support the vitality and differentiation of transplanted chondrocytes $(20,25)$. The disadvantages of periostal flaps are time-consuming technical efforts, the generation of additional cartilaginous lesions by suturing the graft to the cartilage, and the potential for secondary hypertrophy and/or delamination necessitating second-look arthroscopy (16). Moreover, the periosteum exerts potential immunogenic effects (26). Therefore, some attempts were made to replace the periosteum using different resorbable scaffolds (27-33). In our model, the chondrospheres presumably adhered to the cartilage surface border by surface tension forces. Moreover, very good integration results were demonstrated.

The integrative capacity of transplanted chondrospheres was accompanied by the development of an initially more fibroblast-like appearance to a predominating chondrocytic phenotype. This finding is consistent with observations showing a re-differentiation of de-differentiated chondrocytes in high- 
density cultures after culture in monolayers (34) and provides evidence for the chondrogenic potential of chondrospheres.

One limiting factor when comparing our results with ACI in animal joint models is that the influence of mechanical load and kinetic effects on chondrocytes and cartilage in a physiological joint, which are essential for the maintenance of the physiological properties of chondrocytes (35), could not be evaluated. Nevertheless, cartilage-specific matrix proteins such as collagen type II were produced by the chondrospheres in high amounts despite the lack of physiological conditions. Further investigations will have to focus on biomechanical properties of chondrospheres after transplantation as seen under physiological conditions.

In conclusion, these data suggest that human chondrocytes cultured as chondrospheres are able to restore and maintain their phenotype for at least 24 weeks under in vivo conditions in an ACI analogon model. Moreover, chondrospheres adhering to and integrating with superficial cartilage defect surfaces show remarkable remodeling capacity in vivo as shown by an ongoing hyaline-specific maturation of chondrosphere matrix potentially leading to cartilage repair.

\section{Acknowledgements}

The authors would like to thank Mrs. Olga Wiesner and Ms. Carolin Guhr for excellent technical assistance. The study was in part funded by the Co.don AG, Warthestrasse 21, D-14513 Teltow, Germany. The study sponsor was not involved in the collection, analysis and interpretation of data, the writing of the manuscript, or in the decision to submit the manuscript for publication.

\section{Referrences}

1. Ghivizzani SC, Oligino TJ, Robbins PD and Evans CH: Cartilage injury and repair. Phys Med Rehabil Clin N Am 11: 289-307, 2000 .

2. Jorgensen C, Noel D, Apparailly F and Sany J: Stem cells for repair of cartilage and bone: the next challenge in osteoarthritis and rheumatoid arthritis. Ann Rheum Dis 60: 305-309, 2001.

3. Ewing JW and Voto SJ: Arthroscopic surgical management of osteochondritis dissecans of the knee. Arthroscopy 4: 37-40, 1988.

4. Friedman MJ, Berasi CC, Fox JM, Del Pizzo W, Snyder SJ and Ferkel RD: Preliminary results with abrasion arthroplasty in the osteoarthritic knee. Clin Orthop Relat Res 182: 200-205, 1984.

5. Johnson LL: Arthroscopic abrasion arthroplasty historical and pathologic perspective: present status. Arthroscopy 2: 54-69, 1986.

6. Pridie K: Subchondral drilling. J Bone Joint Surg Br 618: 1959.

7. Steadman JR, Rodkey WG and Rodrigo JJ: Microfracture: surgical technique and rehabilitation to treat chondral defects. Clin Orthop Relat Res 391: S362-S369, 2001.

8. Johnson LL: Arthroscopic abrasion arthroplasty: a review. Clin Orthop Relat Res 391: S306-S317, 2001.

9. Brittberg M, Lindahl A, Nilsson A, Ohlsson C, Isaksson O and Peterson L: Treatment of deep cartilage defects in the knee with autologous chondrocyte transplantation. N Engl J Med 331: 889-895, 1994.

10. O'Driscoll SW: Articular cartilage regeneration using periosteum. Clin Orthop 367: S186-S203, 1999.

11. Homminga GN, Bulstra SK, Bouwmeester PS and van der Linden AJ Perichondral grafting for cartilage lesions of the knee. J Bone Joint Surg Br 72: 1003-1007, 1990.

12. Robert $\mathrm{H}$ and Bahuaud $\mathrm{J}$ : Autologous chondrocyte implantation. A review of techniques and preliminary results. Rev Rheum Engl Ed 66: 724-727, 1999.

13. Peterson L: Articular cartilage injuries treated with autologous chondrocyte transplantation in the human knee. Acta Orthop Belg 62: S196-S200, 1996.
14. Schneider U, Breusch SJ and Von Der MK: Current status of autologous chondrocyte transplantation. Z Orthop Ihre Grenzgeb 137: S386-S392, 1999.

15. Muller-Ladner U, Kriegsmann J, Franklin BN, Matsumoto S, Geiler T, Gay RE, et al: Synovial fibroblasts of patients with rheumatoid arthritis attach to and invade normal human cartilage when engrafted into SCID mice. Am J Pathol 149: 1607-1615, 1996.

16. Peterson L, Minas T, Brittberg M, Nilsson A, Sjogren-Jansson E and Lindahl A: Two- to 9-year outcome after autologous chondrocyte transplantation of the knee. Clin Orthop Relat Res 374: 212-234, 2000.

17. Sgaglione NA, Miniaci A, Gillogly SD and Carter TR: Update on advanced surgical techniques in the treatment of traumatic focal articular cartilage lesions in the knee. Arthroscopy 18: 9-32, 2002.

18. Brittberg M, Tallheden T, Sjogren-Jansson B, Lindahl A and Peterson L: Autologous chondrocytes used for articular cartilage repair: an update. Clin Orthop Relat Res 391: S337-S348, 2001.

19. Anderer U and Libera J: In vitro engineering of human autogenous cartilage. J Bone Miner Res 17: 1420-1429, 2002.

20. Litzke LE, Wagner E, Baumgaertner W, Hetzel U, Josimovic-Alasevic O and Libera J: Repair of extensive articular cartilage defects in horses by autologous chondrocyte transplantation. Ann Biomed Eng 32: 57-69, 2004.

21. Giannoni P, Crovace A, Malpeli M, Maggi E, Arbico R, Cancedda $\mathrm{R}$, et al: Species variability in the differentiation potential of in vitro-expanded articular chondrocytes restricts predictive studies on cartilage repair using animal models. Tissue Eng 11: 237-248, 2005.

22. Moretti M, Wendt D, Dickinson SC, Sims TJ, Hollander AP, Kelly DJ, et al: Effects of in vitro preculture on in vivo development of human engineered cartilage in an ectopic model. Tissue Eng 11: 1421-1428, 2005.

23. Song SU, Cha YD, Han JU, Oh IS, Choi KB, Yi Y, et al: Hyaline cartilage regeneration using mixed human chondrocytes and transforming growth factor-beta1-producing chondrocytes. Tissue Eng 11: 1516-1526, 2005.

24. Vangsness CT Jr, Kurzweil PR and Lieberman JR: Restoring articular cartilage in the knee. Am J Orthop 33: S29-S34, 2004.

25. Brittberg M, Sjogren-Jansson E, Thornemo M, Faber B, Tarkowski A, Peterson L, et al: Clonal growth of human articular cartilage and the functional role of the periosteum in chondrogenesis. Osteoarthritis Cartilage 13: 146-153, 2005.

26. Convery FR, Meyers MH and Akeson WH: Fresh osteochondral allografting of the femoral condyle. Clin Orthop Relat Res 273: 139-145, 1991.

27. Haddo O, Mahroof S, Higgs D, David L, Pringle J, Bayliss M, et al: The use of chondrogide membrane in autologous chondrocyte implantation. Knee 11: 51-55, 2004.

28. Benya PD and Shaffer JD: Dedifferentiated chondrocytes reexpress the differentiated collagen phenotype when cultured in agarose gels. Cell 30: 215-224, 1982 .

29. Grande DA, Halberstadt C, Naughton G, Schwartz R and Manji R: Evaluation of matrix scaffolds for tissue engineering of articular cartilage grafts. J Biomed Mater Res 34: 211-220, 1997.

30. Brun P, Abatangelo G, Radice M, Zacchi V, Guidolin D, Daga GD, et al: Chondrocyte aggregation and reorganization into three-dimensional scaffolds. J Biomed Mater Res 46: 337-346, 1999.

31. Nehrer S: Matrix associated chondrocyte transplantation. Biomaterials in cartilage repair. Vavken P DRKR, Editor. Arthroscopy 18: 203-208, 2005.

32. Behrens P, Bitter T, Kurz B and Russlies M: Matrix-associated autologous chondrocyte transplantation/implantation (MACT/ MACI)-5-year follow-up. Knee 13: 194-202, 2006.

33. Krishnan SP, Skinner JA, Carrington RW, Flanagan AM, Briggs TW and Bentley G: Collagen-covered autologous chondrocyte implantation for osteochondritis dissecans of the knee: two- to seven-year results. J Bone Joint Surg Br 88: 203205, 2006.

34. Schulze-Tanzil G, de Souza P, Villegas CH, John T, Merker HJ, Scheid A, et al: Redifferentiation of dedifferentiated human chondrocytes in high-density cultures. Cell Tissue Res 308: 371-379, 2002.

35. Lima EG, Mauck RL, Han SH, Park S, Ng KW, Ateshian GA, et al: Functional tissue engineering of chondral and osteochondral constructs. Biorheology 41: 577-590, 2004. 